\title{
Thomas Fandel
}

\section{Die Stellung von evangelischen und katholischen Pfarrern zum Nationalsozialismus am Beispiel der Pfalz}

„Die katholische und evangelische Entwicklung unterscheiden sich ereignisgeschichtlich und motivisch so stark voneinander, daß es erhebliche Risiken mit sich bringt, sie miteinander zu vergleichen und dabei unter der Hand die Entwicklung der einen Kirche zum Maßstab für die Entwicklung der anderen zu machen. “1 Soweit Kurt Nowak über die Zeit des NS-Regimes in seiner 1995 erschienenen konfessionsübergreifenden "Geschichte des Christentums in Deutschland“. Trotz dieser "Risiken“ hat Nowak selbst das Wagnis einer interkonfessionellen Geschichtsschreibung unternommen und darauf hingewiesen, daß „die Pflege konfessioneller Monokulturen, die den Blick für die Widersprüche, die Wechselspiele und die Identitäten zwischen den beiden großen Konfessionen verstellt, in der

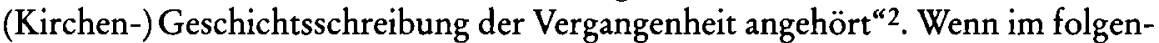
den ein Vergleich des Verhaltens evangelischer und katholischer Pfarrer in der Pfalz im „Dritten Reich“ geboten wird”, soll die zuerst genannte Mahnung Nowaks den Ausführungen ausdrücklich vorangestellt werden. Zwar ist es auch meines Erachtens unerläßlich, beide Konfessionen gemeinsam zu behandeln, um neue Maßstäbe für die nach wie vor umstrittene Bewertung des Verhältnisses der Kirchen zum Nationalsozialismus zu erhalten und um den Blick auf die gegenseitige Beeinflussung der Konfessionen richten zu können. Diese Vorgehensweise darf aber nicht

${ }^{1}$ Kurt Nowak, Geschichte des Christentums in Deutschland. Religion, Politik und Gesellschaft vom Ende der Aufklärung bis zur Mitte des 20. Jahrhunderts (München 1995) 248; vgl. auch Victor Conzemius, Katholische und evangelische Kirchenkampfgeschichtsschreibung im Vergleich: Phasen, Schwerpunkte, Defizite, in: ders. (Hrsg.), Die Zeit nach 1945 als Thema kirchlicher Zeitgeschichte. Referate der internationalen Tagung in Hüningen/Bern (Schweiz) 1985 (Göttingen 1988) 35-57, 51.

2 Nowak, Geschichte $10 \mathrm{f}$.

3 Vgl. Thomas Fandel, Konfession und Nationalsozialismus. Evangelische und katholische Pfarrer in der Pfalz 1930-1939 (VKZG, Reihe B, 76, Paderborn u.a. 1997); dort auch weitere Ausführungen zum Verhältnis von Pfarrern und NSDAP in der Pfalz mit Belegen und Literaturhinweisen. Zur Geschichte der Pfalz in der NS-Zeit vgl. Gerhard Nestler, Hannes Ziegler (Hrsg.), Die Pfalz unterm Hakenkreuz. Eine deutsche Provinz während der nationalsozialistischen Terrorherrschaft (Landau 1993). 
dazu führen, die unterschiedlichen politischen, theologischen und strukturellen Voraussetzungen auszublenden oder gar alte konfessionelle Grabenkämpfe erneut auszufechten, wie dies etwa in der Arbeit von Georg May mit dem programmatischen Titel „Kirchenkampf oder Katholikenverfolgung?" der Fall ist ${ }^{4}$.

Wenn im folgenden von den beiden Kirchen in der Pfalz die Rede ist, dann sind damit das bayerische Bistum Speyer sowie die seit 1818 konsensunierte „Vereinigte Protestantisch-Evangelisch-Christliche Kirche der Pfalz (Pfälzische Landeskirche)"- so die offizielle Bezeichnung - gemeint. Die Grenzen der beiden Kirchen waren identisch. Die Region zeichnete sich durch eine starke konfessionelle Mischung der Bevölkerung aus. 1933 waren 55,75\% der Pfälzer Protestanten, $42,05 \%$ Katholiken, 0,66\% Juden; 1,54\% gehörten anderen religiösen Gemeinschaften an oder waren konfessionslos 5 .

Bis 1920 entsprachen sich in der Pfalz staatliche und kirchliche Grenzen'. Staatsrechtlich gehörte das Gebiet zu Bayern, eine Tatsache, die das politische Bewußtsein der beiden Kirchen auf ganz unterschiedliche Weise prägte, wie noch auszuführen sein wird. Durch den Versailler Vertrag wurden Gebiete im Westen der Pfalz - die sogenannte Saarpfalz - zusammen mit einem Teil des preußischen Regierungsbezirks Trier zu dem dem Völkerbund unterstehenden Saargebiet zusammengefaßt. Im kirchlichen Bereich blieb im Hinblick auf das Saargebiet trotz entgegengesetzter französischer Bemühungen alles beim alten. 1935 sollten die Saarländer über den weiteren völkerrechtlichen Status der Region in einer Volksabstimmung entscheiden - ein Ereignis, das für das Verhältnis der pfälzischen Kirchen zum Nationalsozialismus von erheblicher Bedeutung sein sollte.

Die Phase des „Dritten Reiches“, die im Rahmen dieses Kolloquiums im Blickpunkt steht, war für die Pfarrer der beiden großen christlichen Konfessionen in der Pfalz ein Zeitabschnitt, in dem die Illusionen gründlich zerstört wurden, die man sich teilweise über den Charakter der NS-Herrschaft in Deutschland und insbesondere über die Rolle der Kirchen gemacht hatte. Dies gilt insbesondere für die nicht wenigen evangelischen Theologen, die politische, zum Teil aber auch kirchenpolitische und religiöse Erwartungen mit dem Wirken der NSDAP verbunden hatten. Aber auch für den katholischen Klerus zeigte sich, daß das Entgegenkommen, zu dem sich insbesondere die Bistumsleitung in Speyer bereitgefunden hatte, nicht den gewünschten Widerhall fand. Mit dem erfolgreichen Ausgang der Abstimmung im Saargebiet 1935 endete für die pfälzische Gauleitung die Notwendigkeit, aus taktischen Gründen auf kirchliche Interessen besondere Rücksicht nehmen zu müssen.

${ }^{4}$ Georg May, Kirchenkampf oder Katholikenverfolgung? Ein Beitrag zu dem gegenseitigen Verhältnis von Nationalsozialismus und christlichen Bekenntnissen (Stein am Rhein 1991). Trotz der geäußerten Kritik müssen die von May angeschnittenen Fragen durchaus ernst genommen werden.

5 Statistisches Jahrbuch für Bayern, Bd. 20 (1934) 9.

6 Vgl. Helmut Prantl (Bearb.), Die kirchliche Lage in Bayern nach den Regierungspräsidentenberichten 1933-1943, Bd.V: Regierungsbezirk Pfalz 1933-1940 (VKZG, Reihe A, 24, Mainz 1978) XLI. 
Um die Stellung der pfälzischen Pfarrer zur NSDAP in dem Zeitraum zwischen Herbst 1934 und Herbst 1939 verstehen zu können, sind zunächst einige Bemerkungen über die letzten Jahre der Weimarer Republik sowie die Anfangsphase der nationalsozialistischen Herrschaft nötig.

Die politischen Entwicklungen Ende der zwanziger/Anfang der dreißiger Jahre verschärften die heftigen konfessionellen Gegensätze, die das Leben besonders in den Dörfern der Pfalz prägten. Die immer stärkere Hinwendung vor allem des protestantischen Bevölkerungsteils zur NSDAP bei gleichzeitiger Verurteilung dieser Partei durch die katholische Geistlichkeit ließ die konfessionelle Trennung

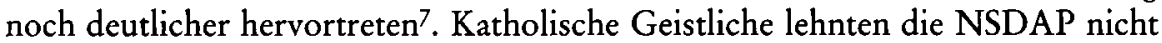
nur aus weltanschaulichen Gründen ab, sondern verstanden sie auch als Bedrohung der durch Zentrum bzw. Bayerische Volkspartei gesicherten gesellschaftlichen Position der Katholiken. Die katholischen Parteien wurden nicht nur von der Kanzel aus unterstützt. Vielfach griffen Geistliche als Funktionäre von Zentrum bzw. Bayerischer Volkspartei direkt in das tagespolitische Geschehen ein. Dabei verschärften nicht wenige Pfarrer auf der lokalen Ebene die differenzierten Anweisungen der Bischöfe, die - gemäß den Kriterien, die auch auf Anhänger von Sozialismus und Liberalismus angewandt worden waren - zwischen NSDAPAgitatoren und bloßen Mitläufern unterschieden.

Während die katholischen Pfarrer in der Regel vorbehaltlos für die katholischen Parteien eintraten und die Mehrzahl der kirchennahen Katholiken dieser Vorgabe folgte ${ }^{8}$, hielten die protestantischen Pfarrer in ihrer überwiegenden Mehrheit Zurückhaltung in parteipolitischen Fragen von Amts wegen für angemessen ${ }^{9}$. Allerdings blieb der Öffentlichkeit nicht verborgen, daß der pfälzische Protestantismus - und mit ihm auch die Pfarrerschaft - immer stärker zur NSDAP tendierte. Gerade im ländlichen Bereich wurde die NSDAP - wie Wolfram Pyta herausgearbeitet hat - immer mehr als protestantische Milieupartei wahrgenommen ${ }^{10}$. Für die Pfarrer waren nicht nur die innen- und außenpoliti-

7 Zur Bedeutung des konfessionellen Faktors für die Wahlerfolge der NSDAP vgl. Jürgen W. Falter, Hitlers Wähler (München 1991), besonders 169-193. Für die Pfalz vgl. u.a. KarlHeinz-Rothenberger, Die nationalsozialistische Machtübernahme in der Südpfalz (Januar November 1933), in: Zeitschrift für die Geschichte des Oberrheins 132 (1984) 305-342, $311 \mathrm{f}$. mit einem Vergleich der Reichstagswahlergebnisse (September 1930) von 16 katholischen (10,5\% NSDAP) und 12 benachbarten protestantischen Gemeinden (63,8\% NSDAP) mit jeweils mehr als $90 \%$ Angehörigen der vorherrschenden Konfession im Bezirk Bergzabern sowie von 10 katholischen $(14,4 \%$ NSDAP) und 6 protestantischen Gemeinden $(51,8 \%$ NSDAP) im Bezirk Landau.

${ }^{8} \mathrm{Daß}$ hinter dieser Entscheidung im allgemeinen kein bewußtes Eintreten für die Weimarer Republik stand, wurde von der Forschung vielfach herausgearbeitet. Vgl. etwa Cornelia Raub-Kübne, Katholisches Milieu und Kleinstadtgesellschaft. Ettlingen 1918-1939 (Geschichte der Stadt Ettlingen 5, Sigmaringen 1991) 422.

${ }_{9}$ Dies belegte u.a. eine entsprechende Unterschriftenaktion des Pfälzischen Pfarrervereins 1931. Vgl. Richard Bergmann (Hrsg.), Documenta. Unsere Pfälzische Landeskirche innerhalb der Deutschen Evangelischen Kirche in den Jahren 1930-1944. Berichte und Dokumente, Bd. 1 (Speyer 1960) $60 \mathrm{f}$.

10 Vgl. Wolfram Pyta, Dorfgemeinschaft und Parteipolitik 1919-1933. Die Verschränkung 
schen Motive von Bedeutung, die das protestantische Bauern- und Bürgertum nach ihrer Abwendung von den in der Pfalz traditionell starken liberalen Parteien in der NSDAP eine neue politische Heimat finden ließen ${ }^{11}$. Hinzu kam die Hoffnung, daß mit Hilfe der NSDAP die Macht des in Bayern - und damit in der Pfalz - besonders einflußreichen politischen Katholizismus gebrochen werden könnte ${ }^{12}$. Eine große Rolle spielte auch die Erwartung, daß das vermeintlich kirchenfreundliche Auftreten der "nationalen Bewegung" die Gelegenheit bieten könnte, kirchendistanzierte Teile der Bevölkerung wieder an die Kirche heranführen zu können und somit dem Ziel „Volkskirche“ näher zu kommen ${ }^{13}$.

Entsprechend der verschiedenartigen Ausgangssituation unterschieden sich die Reaktionen katholischer und protestantischer Pfarrer in der Pfalz auf die politische Umwälzung des Jahres 1933 erheblich. Die geschlossene Teilnahme nationalsozialistischer Gliederungen an den Gottesdiensten, der deutliche Rückgang der Kirchenaustritte ${ }^{14}$, die Wiedereintritte zahlreicher aus der Kirche Ausgetretener ${ }^{15}$ sowie das Verbot kirchenfeindlicher Organisationen schienen die großen Hoffnungen zu bestätigen, die viele Pfarrer der Pfälzischen Landeskirche gehegt hatten. Zwar hinterfragten einige Theologen schon 1933 kritisch die Motivation der vermeintlich aufgebrochenen Kirchlichkeit, doch konnte an dem öffentlichen Prestigegewinn, den die evangelische Kirche zumindest in den ersten Monaten der NS-Herrschaft verbuchen konnte, kein $Z$ weifel bestehen. $\mathrm{Daß}$ die neue politische Führung in die Wahlen zur Landessynode und den Presbyterien eingriff, die 1933 turnusgemäß in der Pfälzischen Landeskirche anstanden, sorgte zwar für erhebliche Irritationen ${ }^{16}$. An der allgemeinen nationalen Euphorie in den Gemeinden konnte dies jedoch nur wenig ändern, zumal in vielen Orten angesichts des Bekenntnisses auch kirchengebundener Protestanten zur NSDAP die Veränderungen in den kirchlichen Gremien eher gering blieben. Lediglich für die in der Pfalz ohnehin sehr wenigen religiös-sozialistischen Pfarrer sowie einige Theologen, die sich als NSDAP-Gegner exponiert hatten, stellte die Machtübernahme der Nationalsozialisten eine Bedrohung ihrer Position in den Gemeinden dar. Ansonsten

von Milieu und Parteien in den protestantischen Landgebieten Deutschlands in der Weimarer Republik (Beiträge zur Geschichte des Parlamentarismus und der politischen Parteien 106, Düsseldorf 1996) 18, 472 u.a.

11 Karl Georg Faber, Überlegungen zu einer Geschichte der Pfälzischen Landeskirche unter dem Nationalsozialismus, in: Blätter für Pfälzische Kirchengeschichte und Religiöse Volkskunde 41 (1974) 29-58, 33 f.

$12 \mathrm{Vgl}$. Fandel, 103-112.

13 Vgl. Pyta, 391-393.

14 Austritte (ohne konfessionsunmündige Kinder) im Bereich der Pfälzischen Landeskirche: 1930-1228, 1931-1103, 1932-1302, 1933-550, 1934-192, 1935-355, 1936-501, 1937-2116, 1938-2130, 1939-1817, 1940-600 (Zentralarchiv der Evangelischen Kirche der Pfalz Speyer im folgenden zitiert: ZASP - Abt. 1 Findbuch 2, 570; zur Interpretation vgl. Fandel, 324332).

15 Wiedereintritte (ohne konfessionsunmündige Kinder) im Bereich der Pfälzischen Landeskirche: 1930-43, 1931-89, 1932-76, 1933-328, 1934-201, 1935-240, 1936-154, 1937-142, 1938-66, 1939-41, 1940-40 (ZASP Abt. 1 Findbuch 2, 570).

16 Vgl. Fandel, 206-212. 
herrschte die Überzeugung vor, daß die evangelische Kirche die NSDAP nur in ihrem Sinne zu beeinflussen brauche, um antichristliche Strömungen innerhalb der Partei zum Verschwinden zu bringen.

Da die Führung der Pfälzischen Landeskirche, die Mehrzahl der Pfarrer und die pfälzische Gauleitung - unbeschadet der heftigen kirchenpolitischen Auseinandersetzungen auf Reichsebene - auf eine harmonische Zusammenarbeit von Protestantismus und Nationalsozialismus setzten, blieben Konflikte zwischen protestantischen Pfarrern und NSDAP in der Pfalz zunächst Ausnahmefälle. Der „Alte Kämpfer" Ludwig Diehl symbolisierte als Landesbischof ab 1934 die weitgehend reibungslose Einbindung der Pfälzer Protestanten in das „Dritte Reich “17. Auch in der Pfarrerschaft fanden sich viele Parteimitglieder; für insgesamt 107 der rund 490 Theologen, die sich zwischen 1933 und 1940 im Dienst der Landeskirche befanden, läßt sich eine Parteimitgliedschaft nachweisen ${ }^{18}$. Das bedeutet, daß etwa jeder fünfte evangelische Pfarrer in der Pfalz NSDAP-Mitglied war, wobei besonders die hohe Zahl junger Parteigenossen auffällt ${ }^{19}$.

Das entscheidende Problem des Jahres 1933 war für die protestantischen Pfarrer - im Gegensatz zum katholischen Klerus - nicht die Bestimmung des Verhältnisses zu den neuen politischen Machthabern. Im Vordergrund stand vielmehr die Ablösung der beiden zuvor in der Pfalz dominierenden kirchenpolitischen Richtungen - des liberalen Protestantenvereins und der konservativen Positiven Vereinigung - durch die Deutschen Christen ${ }^{20}$. Da diese von vielen Pfarrern mehr als Einigungsbewregung innerhalb der Deutschen Evangelischen Kirche denn als nationalsozialistische Kirchenpartei wahrgenommen wurde 21 , schloß sich etwas mehr als die Hälfte aller pfälzischen Theologen der neuen Gruppierung an ${ }^{22}$. Der Konformitätsdruck auf Pfarrer, die sich den neuen politischen und kirchenpolitischen Entwicklungen verschlossen, wurde nicht zuletzt innerkirchlich ausgeübt. Es war bezeichnend, daß der spätere Vorsitzende der Pfälzischen Pfarrbruderschaft, Pfarrer Hans Stempel, sein Amt als Leiter des Predigerseminars in Landau aufgrund des Handelns seiner innerkirchlichen deutsch-christlichen Gegner verlor und nicht durch das Veto von NSDAP-Parteifunktionären. Ganz im Gegen-

17 Zur Biographie Diehls vgl. Hans L. Reichrath, Ludwig Diehl 1894-1982. Kreuz und Hakenkreuz im Leben eines Pfälzer Pfarrers und Landesbischofs (Speyer 1995).

18 Angabe nach Auswertung von Gestapo-Akten (Landesarchiv Speyer H91), kirchlichen Entnazifizierungsakten (besonders ZASP Abt. 160 unverz. Bestand Karton 16 Mappe 108. Namentliche Auflistung von 295 Geistlichen) sowie Auskünften des Berlin Document Center.

19 Von den 107 Parteigenossen unter den pfälzischen Theologen waren beim Parteibeitritt 15 zwischen 17 und 20 Jahren alt, weitere 43 zwischen 21 und 30. Zur Analyse der NSDAPMitglieder in der Pfarrerschaft vgl. Fandel, 506-573.

20 Vgl. Faber, 43-45 sowie Fandel, 212-227.

21 Vgl. Fandel, 217-220, 225.

22 Bis zum November 1933 verzeichnete Diehl als Landesleiter der Deutschen Christen in der Pfalz 177 Mitglieder. Vgl. ZASP Abt. 160,2 Mappe IV und Abt. 160 Nr. 65-67. DCRundschreiben Diehls vom 31. Oktober 1933 (Mitgliederliste), 13. November 1933 und 20. November 1933 (Ergänzungen). Zu diesem Zeitpunkt gab es rund 310 Theologen im Dienst der Pfälzischen Landeskirche. 
teil: Mit dem Landauer NSDAP-Kreisleiter Kleemann fand Stempel sogar einen politischen Fürsprecher ${ }^{23}$.

In auffälligem Kontrast zur protestantischen Hochstimmung stand das Befinden der katholischen Geistlichen nach der Ablösung der BVP-Regierung in Bayern im März 1933. Trotz der Angebote zur Zusammenarbeit, die in der Regierungserklärung Hitlers sowie der darauf erfolgenden Stellungnahme der deutschen Bischöfe von beiden Seiten abgegeben wurden, sah die Situation auf der Gemeindeebene ganz anders aus. Angesichts der unmißverständlichen Ablehnung der NSDAP durch viele Ortsgeistliche vor 1933, die zu erbitterten persönlichen Feindschaften geführt hatte, bestand in nicht wenigen Gemeinden eine hochexplosive Stimmung. Vor allem viele ältere Geistliche machten keinen Hehl daraus, daß sie nicht daran dachten, an ihrem Standpunkt etwas zu ändern. Widerwillig wurde die neue Obrigkeit zwar akzeptiert, an der Ablehnung der NSDAP als solcher aber kein Zweifel gelassen.

Jedoch war dies nur ein Teilaspekt der Entwicklung. Auf der anderen Seite kamen nun die in der Wissenschaft so vieldiskutierten Affinitäten zwischen Nationalsozialismus und Katholizismus ${ }^{24}$ zum Tragen, die es der katholischen Bevölkerung und auch Pfarrern erleichterten, sich mit dem neuen Regime zu arrangieren. Die Bereitschaft der katholischen Kirche, sich einer Mitarbeit unter den neuen Bedingungen nicht zu verschließen, signalisierte in aller Deutlichkeit die Teilnahme vieler Geistlicher an den Feiern zum 1. Mai 1933. In Speyer nahm Bischof Sebastian persönlich mit seinem Generalvikar an dem Aufmarsch teil. Sebastian setzte damit ein Zeichen, das zwar nicht als generelle Zustimmung zum Nationalsozialismus gewertet werden durfte, aber NSDAP-kritischen Katholiken unmißverständlich klarmachte, daß nach den kirchenfreundlichen Äußerungen führender Nationalsozialisten eine Zusammenarbeit nicht an den Katholiken scheitern sollte. Pfarrer, die sich solchen öffentlichen Bekundungen des guten Willens der katholischen Kirche entzogen, gerieten in einen Rechtfertigungszwang gegenüber den Forderungen lokaler NSDAP-Politiker, die auf das Vorbild des Bischofs und anderer Priester verwiesen 25 .

Hoffnungen im katholischen Klerus, durch ein Mitwirken an gesamtgesellschaftlichen Aufgaben das Vertrauen der NSDAP gewinnen zu können, wurden jedoch schnell hinfällig. Durch die Auseinandersetzung um die konfessionellen Volksschulen, die in der Pfalz unmittelbar nach der politischen Umwälzung in Bayern einsetzten, gerieten nicht nur Geistliche, die als entschiedene Gegner der

23 Vgl. Faber, $41 \mathrm{f}$.

24 Vgl. u. a. Ernst-Wolfgang Böckenförde, Der deutsche Katholizismus im Jahre 1933. Kirche und demokratisches Ethos. Mit einem historiographischen Rückblick von Karl-Egon Lönne (Schriften zu Staat, Gesellschaft, Kirche 1, Freiburg, Basel, Wien 1988) sowie Klaus Breuning, Die Vision des Reiches. Deutscher Katholizismus zwischen Demokratie und Diktatur (1929-1934) (München 1969).

25 Vgl. Archiv des Bistums Speyer NA 28/10 Karton 1, Funk. Bericht Funks vom 10. Mai 1946. Nach Darstellung Funks wurde ihm seine Nichtteilnahme an der Feier des 1. Mai 1933 als Lokalkaplan von Edigheim deshalb besonders verübelt, weil alle anderen Geistlichen Ludwigshafens an dem Umzug teilgenommen hatten. 
NSDAP galten, sondern der gesamte Klerus in Bedrängnis. Im Juni 1933 kam es zum Ausbruch der angestauten Aggressionen. In der ganzen Pfalz wurden katholische Geistliche in Schutzhaft genommen, mißhandelt oder zur Flucht aus ihren Pfarreien gezwungen ${ }^{26}$.

Um die Entlassung der Pfarrer aus der Haft zu erreichen, fand sich Bischof Sebastian zu einem Abkommen mit der Gauleitung bereit, in dem den Geistlichen jegliche Kritik an der Regierung Hitler und deren Maßnahmen untersagt wurde ${ }^{27}$. In den folgenden Jahren achtete der Bischof strikt darauf, daß sich sein Klerus jeglicher politischer Äußerung enthielt ${ }^{28}$. Geistliche, denen es darum ging, den Unrechtscharakter des Regimes öffentlich zu machen, waren auf sich allein gestellt. Dem Pfarrer der Gemeinde Rheingönheim bei Ludwigshafen, der aus prinzipiellen Erwägungen nationalsozialistischem Druck auf keinen Fall nachgeben wollte und die Versetzung in eine andere Pfarrei jahrelang verweigerte, nahm der Bischof 1937/38 sogar auf dem Wege eines kanonischen Prozesses seine Pfarrstelle ${ }^{29}$. Für Sebastian hatte die Gewährleistung einer geregelten Seelsorge unbedingten Vorrang, Konfrontationen mit den nationalsozialistischen Machthabern waren zu vermeiden, wenn nicht Kernbereiche kirchlichen Selbstverständnisses angegriffen wurden. Diese „Entpolitisierung des Klerus“ entsprach im übrigen nicht nur den aktuellen politischen Erfordernissen, sondern paßte auch in das Konzept einer "zunehmend gemeindeorientierten und liturgiezentrierten Seelsorge", die für die katholische Kirche in der ersten Hälfte des zwanzigsten Jahrhunderts besonders charakteristisch war ${ }^{30}$.

Nach dem Abschluß des Reichskonkordats, das den Geistlichen ebenfalls jegliche parteipolitische Betätigung untersagte, trat eine gewisse Beruhigung des Verhältnisses zwischen katholischer Kirche und NSDAP in der Pfalz ein. Diese war in erster Linie auf die bevorstehende Abstimmung im Saargebiet zurückzuführen. Gauleiter Bürckel unterband rigoros antikirchliche Bestrebungen und vertagte die Frage der Gemeinschaftsschule bis auf weiteres.

Es war aber nicht nur das Entgegenkommen der pfälzischen NS-Führung, das Bischof Sebastian sowie viele Pfarrer bewog, sich für eine Rückkehr des Saargebietes ins Deutsche Reich einzusetzen. Für die überwiegende Mehrheit der saar-

${ }^{26} \mathrm{Zu}$ regionalen Unterschieden, die nicht zuletzt auf die jeweiligen konfessionellen Bedingungen zurückzuführen waren, vgl. Fandel, 190-203.

27 Vgl. Helmut Prantl, Zur Geschichte der katholischen Kirche in der Pfalz unter nationalsozialistischer Herrschaft, in: Blätter für Pfälzische Kirchengeschichte und Religiöse Volkskunde 42 (1975) 79-117, 87-89.

28 So machte die Kirchenbehörde im Juni 1934 einem Pfarrer in der Südpfalz „sub oboedientia canonica“ zur Auflage, „die Behandlung politischer Verhältnisse bis auf weiteres gänzlich zu unterlassen ". Zitiert nach Helmut Prantl, Geschichte, 95 A. 124.

${ }^{29} \mathrm{Zu}$ den genauen Hintergründen vgl. Fandel, 417-429.

$30 \mathrm{Vgl}$. Priester unter Hitlers Terror. Eine biographische und statistische Erhebung, bearb. von Ulrich von Hebl u. a. (VKZG, Reihe A, 37, Paderborn u. a. ${ }^{31996) ~ B d . ~ 1, ~ 108 . ~ A u c h ~ B i-~}$ schof Sebastian waren allzu exponierte parteipolitische Aktivitäten seiner Seelsorger bereits in der Weimarer Republik ein Dorn im Auge gewesen (vgl. Unterlagen in den Personalakten Dr. Rößler sowie Bisson im Archiv des Bistums Speyer). 
ländischen Katholiken war alles andere als die Option für Deutschland undenkbar, wäre als nationaler Verrat empfunden worden. Um so bemerkenswerter - und bezeichnend für das Verhältnis von katholischen Geistlichen und NS-Regime - ist es, daß dennoch eine nicht unerhebliche Zahl von Pfarrern ${ }^{31}$ unter den gegebenen Bedingungen gegen den Anschluß der Region an Deutschland eingestellt war, da sie nicht in das "Dritte Reich" eingegliedert werden wollten. Für sechs Geistliche aus den Pfarreien des Saargebietes, die zur Diözese Speyer gehörten, war der Ausgang der Abstimmung sogar Anlaß, sich ins Exil zu begeben ${ }^{32}$. Daß nicht ihre Haltung, sondern die der Bischöfe Sebastian und Bornewasser, die politische Zurückhaltung, zugleich aber eine klare nationale Haltung der Priester gefordert hatten, der Stimmung der katholischen Bevölkerung entsprach, zeigte das Abstimmungsergebnis: Die mehrheitlich katholischen Saarländer entschieden sich mit einer überwältigenden Mehrheit von 90,73 Prozent der Stimmen für eine Rückkehr in das Deutsche Reich ${ }^{33}$.

Im Gegensatz zur katholischen Kirche bereitete es der Pfälzischen Landeskirche keine Schwierigkeiten, daß die Rückkehr des Saargebietes in das Deutsche Reich die Eingliederung in ein nationalsozialistisches Deutschland bedeutete. Landesbischof Diehl konnte in einer Predigt zum 1. März 1935 befriedigt die „gerade Haltung“ der protestantischen Gemeinden rühmen, die "mit ihren Pfarrern sich für die baldige Rückkehr zum Reiche Adolf Hitlers" eingesetzt hatten ${ }^{34}$. Auch in den Jahresberichten der Pfarrer klang Stolz an, daß die nationale Zuverlässigkeit der Protestanten nicht - wie beim katholischen Bevölkerungsteil - in Zweifel gezogen worden war ${ }^{35}$.

Nach der Abstimmung mußten jedoch auch die evangelischen Pfarrer erkennen, daß die vermeintliche Wertschätzung ihrer Kirche lediglich auf zeitweilige politische Notwendigkeiten zurückzuführen gewesen war. Im Jahresbericht der Gemeinde Hassel bei St. Ingbert für das Jahr 1935 hieß es beispielsweise: „Während man in der Zeit des Abstimmungskampfes die Kirche bei jedem Anlaß brauchte, nimmt man heute keine Rücksicht mehr auf sie. Am Erntedankfest 1934 ordneten die Führer der Deutschen Front (die jetzigen Führer der Ortsgruppe der

31 Mindestens ein Drittel der Geistlichen, die in den 35 zur Diözese Speyer zählenden Pfarreien im Saargebiet tätig waren, verhielt sich so, daß sie von den Vertretern der Deutschen Front scharf angegriffen wurden (vgl. Fandel, 246-258).

32 Vgl. Archiv des Bistums Speyer. Personalakten Ecker, Engesser, Franz, Mühl, Pfeiffer, Weber; vgl. auch Kurt Schöndorf, „Für Christus und Deutschland - Gegen Hitler und die Neuheiden". Zum Widerstand katholischer Geistlicher gegen Hitler in der Saarpfalz vor der Saarabstimmung 1935, in: Saarpfalz 1994/2, 25-34; Gerbard Paul, Klaus-Michael Mallmann, Milieus und Widerstand. Eine Verhaltensgeschichte der Gesellschaft im Nationalsozialismus (Widerstand und Verweigerung im Saarland 1935-1945, 3, Bonn 1995) 80.

33 Abdruck der Wahlergebnisse bei Markus Gestier, Die christlichen Parteien an der Saar und ihr Verhältnis zum deutschen Nationalstaat in den Abstimmungskämpfen 1935 und 1955 (Saarbrücker Hochschulschriften 15, St. Ingbert 1991) Anlage 42, $82 \mathrm{f}$.

34 ZASP Abt. 44/St. Ingbert Nr. 17b. Jahresbericht Pfarrer Kaisers für St. Ingbert 1935.

35 Vgl. z. B. ZASP Abt. 8 Nr. 73. Jahresbericht Pfarrer Foells für Homburg 1934. 
NSDAP) geschlossenen Kirchgang an, am Erntedankfest 1935 exerzierte während des Gottesdienstes die SA auf dem hiesigen Sportplatz."36

Wahrnehmungen wie diese bestimmten in der Folgezeit zunehmend das Verhältnis von evangelischen Pfarrern und NSDAP innerhalb des gesamten Gebietes der Pfälzischen Landeskirche. Die Pfarrer mußten registrieren, daß die Gleichschaltung der Landeskirche von der NSDAP nicht mit der erhofften Wertschätzung honoriert wurde. Hatte etwa die Landessynode im März 1934 beschlossen, die Zugehörigkeit zu SA, SS oder einer anderen NS-Organisation zur Voraussetzung zur Zulassung zum theologischen Examen zu machen ${ }^{37}$, so wurde diese Auflage schnell hinfällig, weil Theologen in der Partei und deren Gliederungen nicht mehr erwünscht waren. Wenn viele evangelische Theologen in der Pfalz trotz dieser offenen Brüskierung weiterhin von einer grundlegenden Vereinbarkeit von Nationalsozialismus und Christentum ausgingen, hing dies nicht zuletzt mit der kirchenpolitischen Linie der pfälzischen Gauleitung zusammen. Neuheidnische Bestrebungen einzelner Nationalsozialisten in pfälzischen Städten und Dörfern fanden die ausdrückliche Mißbilligung der Gauleitung ${ }^{38}$. Ebenso wurden in Einzelfällen von lokalen Parteifunktionären erzwungene Kirchenaustritte rückgängig gemacht ${ }^{39}$. Die Gauleitung konnte kein Interesse daran haben, vom Nationalsozialismus überzeugte Protestanten gegen sich aufzubringen oder durch offen ausgetragene kirchenpolitische Streitigkeiten Unruhe in die Bevölkerung hineintragen zu lassen. Zudem waren führende pfälzische Nationalsozialisten wie der stellvertretende Gauleiter Leyser ${ }^{40}$ oder der Regierungsdirektor im Reichskommissariat für das Saarland, Barth, den Bürckel aus dem Dienst der Landeskirche übernommen hatte, gläubige Protestanten.

Erst durch den zunehmenden Einfluß der Gestapo ab 1937 und verstärkte Kirchenaustritte von Parteifunktionären auch in der Pfalz wurde den evangelischen Pfarrern endgültig klar, daß nicht die Linie der pfälzischen Gauleitung maßgeblich für die kirchenpolitische und weltanschauliche Entwicklung in Deutschland war. Verfolgungsmaßnahmen ${ }^{41}$, die sich bisher vor allem auf katholische Geist-

36 ZASP Abt. 8 Nr. 71. Jahresbericht Pfarrer Oberlingers für Hassel 1935, ähnlich der Jahresbericht für 1936.

37 Verhandlungen der Landessynode der Vereinigten Protestantisch-Evangelisch-Christlichen Kirche der Pfalz in den Jahren 1930-1944 (Grünstadt 1959) 408f., 415 f., 421, 426 f., $434 \mathrm{f}$.

38 Vgl. die Belege bei Prantl, Kirchliche Lage, Register „Glaubensbewegung, Deutsche“, z.B. 76 .

39 Vgl. Bergmann, Bd. 3, 77-82.

$40 \mathrm{Zu}$ Leyser vgl. Dieter Wolfanger, Ernst Ludwig Leyser. Stellvertretender Gauleiter der NSDAP in der Saarpfalz. Eine biographische Skizze, in: Jahrbuch für westdeutsche Landesgeschichte 14 (1988) 209-217.

41 Vgl. Fandel, 399-465; Priester unter Hitlers Terror Bd. 2, 1363-1420; Karl Heinz Debus, Verfolgung und Widerstand in der Pfalz. Ethnische Minderheiten und Religionsgemeinschaften unter nationalsozialistischem Terror. Eine topo-prosopographische Studie, in: Palatia Historica. Festschrift für Ludwig Anton Doll zum 75. Geburtstag, hrsg. von Pirmin Spieß (Quellen und Abhandlungen zur mittelrheinischen Kirchengeschichte 75, Mainz 1994) $627-723$. 
liche bezogen hatten und diese auch weiterhin viel stärker betrafen, wurden nun auch für evangelische Pfarrer nicht mehr nur in Einzelfällen zu einem Thema. Dies galt selbst für Pfarrer, die wie der Kaiserslauterer Pfarrer Hans Schmidt in den Gestapo-Akten als anerkannte "Vorkämpfer der nationalen Erhebung“42 eingestuft wurden. Verbitterung machte sich gerade bei jenen protestantischen Pfarrern breit, die nach wie vor überzeugte Nationalsozialisten waren. Der Oppauer Pfarrer Stöppler etwa, der in seinen Jahresberichten die nationalsozialistische Regierung teilweise euphorisch gelobt hatte ${ }^{43}$, schrieb 1938 in einem Brief an Landesbischof Diehl: „Unsere Gemeinde, unsere Protestanten, die rechtschaffene Nationalsozialisten gewesen sind, die fühlen sich verraten und belogen, die fühlen sich in ihrem Glauben aufs schlimmste enttäuscht!" 44 Stöppler zählte zu jenen Parteigenossen im Talar, die angesichts des wachsenden Einflusses kirchenfeindlicher Tendenzen in Partei und Gesellschaft dafür eintraten, daß die evangelische Kirche durch besondere nationalsozialistische Prinzipientreue ihre Loyalität unter Beweis stellen müsse. Andere Pfarrer - darunter auch Parteigenossen - zogen ganz andere Konsequenzen. Vor allem jüngere Theologen gingen unter dem Einfluß der Barmer Theologie sowie der faktischen Entwicklung der Partei auf Distanz zur NSDAP, zumindest im Hinblick auf deren Kirchenpolitik.

Daß es sich nur in Einzelfällen um eine grundlegende Umstellung der bisherigen politischen Linie der evangelischen Pfarrer handelte, verdeutlicht ein Blick auf die Vertreter der Bekennenden Kirche in der pfälzischen Pfarrerschaft. Im September 1934 - nach der Eingliederung der Pfälzischen Landeskirche in die Deutsche Evangelische Kirche - fanden sich Pfarrer verschiedener kirchenpolitischer Richtungen zusammen, um sich zur Pfälzischen Pfarrbruderschaft ${ }^{45}$ zusammenzuschließen. Die Pfarrbruderschaft, die im August 1938 rund 29 Prozent der Pfarrerschaft in ihren Reihen vereinigte ${ }^{46}$, war - ebenso wie die Bekennende Kirche in anderen Landeskirchen ${ }^{47}$ - keineswegs eine wie auch immer geartete politische Opposition gegen das NS-Regime. Ziel war vielmehr ein koordiniertes Auftreten gegen das deutsch-christliche Kirchenregiment von Landesbischof Diehl. Bereits

42 Landesarchiv Speyer H91/1356. Schreiben der Kriminalpolizei Kaiserslautern an die Gestapo Neustadt vom 18. März 1938.

43 ZASP Abt. 8 Nr. 191.

${ }_{44}$ Zitiert nach Bergmann, Bd. 3, 83. Zu Stöppler sowie Oppau, wo bereits in der Weimarer Republik starke antikirchliche Aktivitäten zu verzeichnen waren, vgl. Fandel, 79f., 547-553. 45 Zur Pfälzischen Pfarrbruderschaft vgl. Fandel, 265-293.

46 Vgl. die Mitgliederliste aus dem Privatnachlaß von Pfarrer Wilhelmy bei Walter Becker, Entschlossenes Bekenntnis im III. Reich innerhalb der Pfälzischen Landeskirche, dargestellt am Beispiel des Pfarrers Heinz Wilhelmy, Wissenschaftliche Zulassungsarbeit zur 1. Theologischen Prüfung bei der Evangelischen Kirche der Pfalz (Protestantische Landeskirche) (Heidelberg 1986) (Exemplar der ungedruckten Arbeit im ZASP) Anhang 51 sowie eine Mitgliederliste aus dem Nachlaß Pfarrer Wiens (ZASP Abt. 150 Nr. 40a).

47 Vgl. z.B. Karl-Ludwig Sommer, Bekenntnisgemeinschaft und bekennende Gemeinden in Oldenburg in den Jahren der nationalsozialistischen Herrschaft. Evangelische Kirchlichkeit und nationalsozialistischer Alltag in einer ländlichen Region (Veröffentlichungen der historischen Kommission für Niedersachsen und Bremen 39: Niedersachsen 1933-1945, 5, Hannover 1993). 
unmittelbar nach der Gründung der Pfarrbruderschaft kam es zu einer Verständigung aller Kräfte innerhalb der Landeskirche, wonach im Hinblick auf die Saarabstimmung keine kirchenpolitischen Auseinandersetzungen in die Gemeinden getragen werden sollten. Bis zum Auftreten der nationalkirchlichen Bewegung in der Pfalz 1937 informierten die meisten protestantischen Pfarrer ihre Gemeinden weder eingehend über den Streit innerhalb der Deutschen Evangelischen Kirche noch über die sich neu bildenden Fronten in der Pfalz.

Wie wenig es der Pfälzischen Pfarrbruderschaft um eine oppositionelle Haltung zu Staat und Partei ging, zeigt auch die Bereitschaft, 1936 in die deutsch-christlich dominierte Kirchenregierung einzutreten. Im gleichen Jahr lieferte die überwältigende Mehrheit der Pfarrbrüder einen vom Landeskirchenrat geforderten Nachweis der „arischen“ Abstammung der Pfarrer und ihrer Ehefrauen. Nur zehn, zumeist jüngere Mitglieder der Pfarrbruderschaft verweigerten diesen Nachweis, den sie innerhalb der Kirche - nicht innerhalb des staatlichen Rahmens - ablehnten $^{48}$. Auch in der Frage des Treueides auf Hitler im Jahr 1938 gab es in der Pfalz nur ganz wenige Verweigerer, die sich schon zuvor von der Pfarrbruderschaft wegen deren Kompromißbereitschaft gelöst hatten.

Die Regierung der Pfalz erkannte die Loyalität der Pfälzischen Landeskirche ausdrücklich an und wies in ihrem Monatsbericht vom 7. September 1937 auf die Problematik zentral angeordneter Überwachungsmaßnahmen hin, die für die Pfalz als kontrapunktiv angesehen wurden: „Die Notwendigkeit einer Überwachung der katholischen Predigten wird auch für die Zukunft anerkannt werden müssen; indes ist die Überwachung der Erklärungen evangelischer Geistlicher, die im Regierungsbezirk Pfalz in der Mehrzahl dem Nationalsozialismus positiv gegenüberstehen, vielfach geeignet, bisher loyale Geistliche in einen Zustand der Renitenz zu versetzen. Da die Pfalz von dem Kirchenkampf innerhalb der evangelischen Kirche bisher noch ziemlich verschont geblieben ist, treffen die von zentraler Stelle angeordneten Überwachungsmaßnahmen vielfach nicht auf die pfälzischen Verhältnisse zu, wie ja auch die Ergebnislosigkeit der meisten Überwachungen bestätigt. Es dürfte an der Zeit sein, in der Überwachung der evangelischen Gottesdienste eine Milderung eintreten zu lassen und diese nur auf bekannte Hetzer zu beschränken. " 49

Ein Blick auf das Jahr 1937, in dem der Bericht verfaßt wurde, dokumentiert trotz der zunehmenden Aktivitäten der Gestapo noch einmal, wie sehr die Zeit des „Dritten Reiches“ für die evangelischen Pfarrer in der Pfalz von innerkirchlichen Auseinandersetzungen bestimmt wurde. Durch das Auftreten der nationalkirchlichen Bewegung sahen sich Deutsche Christen, die Pfälzische Pfarrbruderschaft und die liberale Vereinigung „Freunde der Union" veranlaßt, sich gemeinsam in Sondergottesdiensten zu ihrem angestammten Glauben zu bekennen ${ }^{50}$.

48 An dem Recht des Staates zur Forderung des Ariernachweises ließen auch fast alle der Verweigerer keinen Zweifel (vgl. die Unterlagen im ZASP Abt. 160,3 Bd. 1 Nr. 3).

49 Prantl, Kirchliche Lage 204.

50 Zur nationalkirchlichen Bewegung in der Pfalz vgl. Fandel, 333-398. 
Zwar warb im Bereich der Pfälzischen Landeskirche nur etwa ein Dutzend Theologen für die konfessionelle Vereinigung der Deutschen unter völkischen Vorzeichen, doch kam es in den betroffenen Orten - vor allem in Städten wie Speyer, Ludwigshafen, Frankenthal und Neustadt - zu regelrechten Spaltungen der Gemeinden. Obwohl gerade von Nationalkirchlern mit politischen Verdächtigungen gearbeitet wurde, handelte es sich nicht um eine politische, sondern um eine kirchenpolitische Angelegenheit. Die Nationalkirchler erhielten - von Ausnahmen auf lokaler Ebene abgesehen - keineswegs die Unterstützung der NSDAP51. Mit dem Speyerer Pfarrer Emil Lind betätigte sich ein Theologe führend für die $\mathrm{Na}$ tionalkirche, der als NSDAP-Gegner galt und sich 1933 als erster und zunächst auch einziger pfälzischer Pfarrer dem Pfarrernotbund angeschlossen hatte. Lind erhoffte sich mittels der nationalkirchlichen Bewegung nicht die Durchsetzung politischer Maxime, sondern die Umsetzung seiner liberalen Theologie in einer Personalgemeinde 52 .

Auch wenn sich in allen kirchenpolitischen Gruppierungen Parteigenossen engagierten, muß - im Gegensatz zu Untersuchungen aus anderen Landeskirchen ${ }^{53}$ betont werden, daß sich bei den Pfarrern der Pfälzischen Landeskirche durchaus eine Beziehung zwischen NSDAP-Parteimitgliedschaft und kirchenpolitischem Bekenntnis feststellen läßt. Die Deutschen Christen und vor allem die nationalkirchliche Bewegung hatten - prozentual gesehen - wesentlich mehr Parteimitglieder aufzuweisen als die Anhänger der Bekennenden Kirche ${ }^{54}$.

Während sich die evangelischen Pfarrer 1937 vor allem mit ihren nationalkirchlichen Kollegen auseinanderzusetzen hatten, war dieses Jahr für die katholischen Pfarrer vor allem durch erneute Auseinandersetzungen um die konfessionellen Schulen geprägt. So sehr Bischof Sebastian in politischen Fragen auf Zurückhaltung seines Klerus drang, so sehr war er gleichzeitig darauf bedacht, daß die Priester in Fragen, in denen er Belange der Kirche unmittelbar berührt sah, ohne Rücksicht auf persönliche Risiken den Standpunkt der Kirche vertraten. Abgesehen von wenigen Ausnahmen konnte sich Sebastian auf seinen Klerus verlassen, der ohnehin zu einem nicht geringen Teil dem diplomatisch-taktierenden Kurs des Bischofs mit großen Vorbehalten gegenüberstand. Ende 1936/Anfang 1937 wurde die katholische Bevölkerung auf den zu erwartenden Angriff auf das konfessionelle Volksschulsystem vor allem durch Predigten vorbereitet. Zusätzliche Brisanz erhielt die Schulfrage durch eine lokale Auseinandersetzung um den Rang

51 Vgl. Fandel, 386 f., 374.

$52 \mathrm{Zu}$ Lind vgl. Fandel, 346-368.

53 Clemens Vollnhals, Die Hypothek des Nationalprotestantismus. Entnazifizierung und Strafverfolgung von NS-Verbrechen nach 1945, in: Geschichte und Gesellschaft 18 (1992) 51-69, 57 sowie Rainer Lächele, Ein Volk, ein Reich, ein Glaube. Die „Deutschen Christen“ in Württemberg 1925-1960 (Quellen und Forschungen zur Württembergischen Kirchengeschichte 12, Stuttgart 1994) 229.

$54 \mathrm{Vgl}$. Fandel, 342f., 521-523. Von den 13 ermittelten nationalkirchlichen Theologen waren sechs NSDAP-Mitglied. Dagegen fanden sich unter den gut 100 Mitgliedern der Pfälzischen Pfarrbruderschaft lediglich 16 Parteigenossen; von diesen wurden drei aus der NSDAP ausgeschlossen, sechs traten aus. 
von Hitlerbildern und Kreuzen, den sogenannten Frankenholzer Schulkreuzstreit ${ }^{55}$. Der Schulstreik in der saarpfälzischen Gemeinde war zwar - worauf Gerhard Paul hingewiesen hat - kein "Akt katholischen Widerstandes" ${ }^{56}$, der eine Aufkündigung politischer Loyalitäten anzeigte, dennoch trafen seine Auswirkungen Gauleiter Bürckel an einem äußerst empfindlichen Punkt. Bischof Sebastian machte den Vorfall in der ganzen Diözese publik und ließ keinen Zweifel daran bestehen, daß Frankenholz eben kein Einzelfall, sondern vielmehr symptomatisch für die Gesamtentwicklung im Deutschen Reich war, die darauf hinauslief, die christlichen Kirchen durch eine neue "deutsche Kirche" zu ersetzen ${ }^{57}$.

Zwar gelang dem Gauleiter mit der erfolgreichen Durchführung der Abstimmung über die Einführung der Gemeinschaftsschule am 20. März 1937 ein auch propagandistisch zu verwertender Sieg über die katholische Kirche ${ }^{58}$. Das Abstimmungsergebnis zeigte trotz allen Drucks und aller Manipulationen, daß die Bereitschaft der meisten Katholiken, sich Forderungen der NSDAP zu widersetzen, dort an ihre Grenze stieß, wo konkrete Nachteile - vor allem wirtschaftlicher Art - drohten. Die klaren Worte des Bischofs ließen jedoch die von Bürckel "gepflegte Fiktion des ,Religionsfriedens" 59 in seinem Gau endgültig in sich zusammenbrechen. Hinzu kam, daß die bischöfliche Kritik durch die Verlesung der Enzyklika "Mit brennender Sorge“ am Tag nach der Schulabstimmung aus katholischer Sicht mit höchster Autorität bestätigt wurde. Der Gauleiter, der selbst aus einer fest im katholischen Milieu verankerten Familie stammte, legte es nun darauf an, Sebastian, der ihm in der Frage des Saargebietes von so großem Nutzen gewesen war, öffentlich zu diskreditieren und mit ihm die katholische Kirche als überholte und national unzuverlässige Institution darzustellen ${ }^{60}$.

Die Auseinandersetzungen um die Schulen, die aufgrund der liberalen Tradition der Landeskirche im protestantischen Bereich kein Pendant fanden, lassen die Eigenart des Verhältnisses von katholischen Pfarrern und NSDAP klar hervortreten. Es ging nicht um eine politische Opposition gegen das NS-Regime, sondern um „weltanschauliche Immunisierung und kirchlich institutionelle Selbstbehauptung "61. Die Auseinandersetzung wurde gerade in der Schulfrage mit besonderer Heftigkeit geführt, weil beide Seiten eine Erziehung nachfolgender Generationen in ihrem Sinne gewährleisten wollten. Auch in der Jugendarbeit verteidigte die katholische Kirche zäh ihre Eigenständigkeit, während die evangelische Kirche in

55 Vgl. Paul, Mallmann, 129-134 sowie Fandel, 299-306.

56 Klaus-Michael Mallmann, Gerbard Paul, Resistenz oder loyale Widerwilligkeit? Anmerkungen zu einem umstrittenen Begriff, in: Zeitschrift für Geschichtswissenschaft 41 (1993) 129.

57 Vgl. den Hirtenbrief Sebastians, unterzeichnet am 28. Februar 1937, verlesen am 7. März 1937, unter Bezugnahme auf Rosenberg; Abdruck bei Jakob Bisson, Sieben Speyerer Bischöfe und ihre Zeit. 1870 bis 1950. Beiträge zur heimatlichen Kirchengeschichte (Speyer 1956) 348-351, Zitat 348.

$58 \mathrm{Zu}$ der Abstimmung vgl. Fandel, 306-318.

59 Prantl, Geschichte 112.

$60 \mathrm{Vgl}$. Prantl, Geschichte $112 \mathrm{f}$.

61 Priester unter Hitlers Terror Bd. 1, 115. 
die Eingliederung ihrer Jugend in die HJ einwilligte und sich in der Folgezeit äußerst schwertat, Jugendliche in einem genuin kirchlichen Rahmen zusammenzuführen ${ }^{62}$. In ländlichen Gemeinden gelang es katholischen Pfarrern sogar teilweise, den nationalsozialistischen Jugendorganisationen das Wasser abzugraben und Kinder und Jugendliche für entsprechende katholische Organisationen zu gewinnen ${ }^{63}$. Nicht nur im Hinblick auf den Nachwuchs hatten die katholischen Pfarrer, die traditionell im katholischen Milieu nicht nur in rein kirchlichen Fragen die Meinungsführerschaft besaßen, wesentlich größere Möglichkeiten, die Bevölkerung in ihrem Sinne zu beeinflussen, als ihre evangelischen Kollegen. Zwar bezog sich die Abgrenzung zur NSDAP in erster Linie auf kirchliche Eigeninteressen ${ }^{64}$, doch bewirkte das Beharren auf einer eigenständigen Tradition - die Teilabschottung von gesamtgesellschaftlichen Entwicklungen im „Dritten Reich“ - eine Immunisierung gegenüber dem nationalsozialistischen Totalitätsanspruch.

Die intendierte völlige weltanschauliche Durchdringung der Gesamtgesellschaft mußte im Hinblick auf die kirchennahen Katholiken scheitern, die - angeleitet von ihren Geistlichen - nicht bereit waren, die neue nationalsozialistische Weltsicht mit ihrer Verabsolutierung des „deutschen“ Menschen zu akzeptieren. Insofern erscheint der von Broszat eingeführte Begriff der Resistenz $z^{65}$ - trotz aller daran geäußerter und zum Teil auch berechtigter Kritik ${ }^{66}$ - geeignet, die spezifische Eigenart des Verhaltens katholischer Kleriker und der sie stützenden katholischen Kerngemeinden zu beschreiben, wenn zugleich eine klare Abgrenzung zu einem Widerstands-Begriff vorgenommen wird, der Handlungen beschreibt, die auf einen Umsturz des NS-Regimes abzielten, und die Gemengelage von Teilablehnung und Teilzustimmung nicht übersehen wird. Mit Cornelia Rauh-Kühne muß zudem ausdrücklich vor einer Überbewertung der "Resistenz" im katholischen Kirchenvolk vor allem in Fragen gewarnt werden, die sich nicht unmittelbar auf Kirche und Religion bezogen ${ }^{67}$.

„Widerstand“, der eine Aufkündigung der politischen Loyalität beinhaltete, wurde auch nicht von jenen katholischen Geistlichen geleistet, die gegenüber den Machthabern eine kämpferischere Linie für richtig hielten, als sie von Bischof Sebastian bevorzugt wurde. In dieser Hinsicht gab es durchaus Parallelen zwischen katholischen Geistlichen und jenen evangelischen Pfarrern, die den eindeutig pro-nationalsozialistischen Kurs ihrer Kirchenleitung nicht teilten und ihre Gemeinden entschlossen auf die Verteidigung ihres Glaubens vorbereiteten. Auch fehlte selbst bei diesen Pfarrern - bis auf ganz wenige Ausnahmen - konfessionsübergreifend die Bereitschaft, sich über die eigenen Grenzen hinaus für Verfolgte

$62 \mathrm{Vgl}$. Fandel, $323 \mathrm{f}$.

63 Vgl. Monatsbericht der Regierung der Pfalz vom 8. Juni 1935 (Prantl, Kirchliche Lage 74).

64 Paul, Mallmann, 116-135, $533 \mathrm{f}$.

65 Martin Broszat, Resistenz und Widerstand. Eine Zwischenbilanz des Forschungsprojekts, in: Bayern in der NS-Zeit, Bd.4, hrsg. von Martin Broszat u.a. (München, Wien 1981) 691-709.

66 Vgl. u. a. Paul, Mallmann, 99-116.

67 Cornelia Raub-Kühne, 389 A. 346 
einzusetzen. Die Segmentierung der deutschen Gesellschaft in weitgehend geschlossene Milieus bedingte eine Blickverengung auf die jeweils eigenen Interessen. So blieb auch die unmißverständliche Stellungnahme des evangelischen Pfarrers von Mutterstadt gegen die Vorfälle in der Reichspogromnacht 1938 eine Ausnahme ${ }^{68}$. Die von Ian Kershaw getroffenen Feststellungen über die Haltung von bayerischen Pfarrern beider Konfessionen zur nationalsozialistischen Judenverfolgung treffen auch für die Pfalz zu' ${ }^{69}$. Die meisten Pfarrer schwiegen und sahen keinen Anlaß, sich in einer Frage zu exponieren, die ihnen angesichts der Bedrohung kirchlicher Belange und der Gefährdung der eigenen Person von nachgeordneter Bedeutung erschien. Eine gewichtige Rolle spielten darüber hinaus Vorbehalte gegenüber den Juden, die sowohl aus traditionellem christlichen Antijudaismus als auch aus „sittlich-soziokultureller Judenfeindschaft ${ }^{\text {“70 }}$ gespeist wurden.

Trotz der konfessionellen Gemeinsamkeiten sind auch in dieser Frage Unterschiede zwischen evangelischen und katholischen Pfarrern in der Pfalz festzustellen. Rassenfanatiker im Sinne der NS-Ideologie gab es zwar selbst unter den evangelischen Pfarrern, die NSDAP-Mitglied waren, nur wenige. Allerdings war es nicht zuletzt auf ihr Wirken zurückführen, daß es innerhalb der Pfälzischen Landeskirche zu Diskussionen kam, die im katholischen Bereich keine Parallele fanden. So stellte 1939 der nationalkirchliche Pfarrer Willmann in einer Sitzung der Kirchenregierung den Antrag, daß Juden nicht Mitglieder der Landeskirche sein könnten. Der Antrag wurde zwar abgelehnt, jedoch erklärte Oberkirchenrat Stichter, um die Kirchenregierung vor politischen Verdächtigungen durch die Nationalkirchler zu schützen, „es dürfe nach diesem Abstimmungsergebnis nicht die Ansicht entstehen oder verbreitet werden, als ob diejenigen Mitglieder, die nicht für den Antrag gestimmt haben, wünschten, daß Juden Mitglieder der Pfälzischen Landeskirche werden sollen"71. Auch an der Basis zeigte die NS-Ideologie Auswirkungen: In Speyer zogen 1936 drei jüdische Taufbewerber ihr Gesuch wieder zurück, nachdem Gemeindemitglieder mit ihrem Kirchenaustritt gedroht hatten ${ }^{72}$.

Das Verhältnis zu den Juden war für die pfälzischen Pfarrer beider Konfessionen im „Dritten Reich“ eher ein Randproblem. Im Vordergrund stand jeweils die Wahrung der eigenen Interessen, die von evangelischer und katholischer Seite

68 Nachdem am 10. November 1938 die Synagoge in Mutterstadt (Bezirk Ludwigshafen) in Brand gesteckt worden war, hatte Pfarrer Bähr im Religionsunterricht gesagt: „Was hier gemacht wird, ist nicht recht - Juden sind auch Menschen und mit Menschen muß man immer menschlich umgehen." Bähr wurde daraufhin festgenommen, jedoch auf Intervention von Landesbischof Diehl zwei Tage später wieder freigelassen. Vgl. Bergmann, Bd. 3, 45.

${ }^{69} \mathrm{Vgl}$. Ian Kershaw, Popular Opinion and Political Dissent in the Third Reich: Bavaria 1933-1945 (Oxford 1983) 246-257.

70 Heinz Eduard Tödt, Das Novemberverbrechen 1938 und der deutsche Protestantismus. Ideologische und theologische Voraussetzungen für die Hinnahme des Pogroms, in: Kirchliche Zeitgeschichte 2 (1989) 14-37, Zitat 31.

71 Zitiert nach Reichrath, 56.

72 Eberhard Röbm, Jörg Thierfelder, Juden, Deutsche, Christen 1933-1945, Bd. 2/I (Stuttgart 1992) 74-77. 
unterschiedlich definiert wurden. Auf Grund der politischen Nähe vieler protestantischer Pfarrer in der Pfalz zum Nationalsozialismus, aber auch auf Grund eines anderen Verständnisses von der Rolle der Kirche in der Gesellschaft ergab sich ein wesentlich geringeres Konfliktpotential zwischen protestantischen Pfarrern und Nationalsozialisten, als es zwischen katholischen Pfarrern und Nationalsozialisten bestand. Die katholische Form des Christentums machte generell die Anpassung an staatliche Vorgaben schwerer als die evangelische - unabhängig davon, um welche Staatsform es sich handelte. Die Konfliktfelder waren zum Teil in der Weimarer Republik und im „Dritten Reich“ dieselben - genannt sei nur die Frage der Konfessionsschulen ${ }^{73}$. In einem totalitären Staat mußten die katholischen Einwände gegen Modernisierungs- und Säkularisierungstendenzen ${ }^{74} \mathrm{je}^{-}$ doch auf besonders heftige Gegenreaktionen stoßen, da dieser das katholische Beharren auf einer eigenständigen Tradition auf keinen Fall hinnehmen wollte und mit Gewalt bekämpfte.

Die katholischen Pfarrer sahen sich durch ihren Bischof und das Reichskonkordat zur politischen Zurückhaltung verpflichtet, zur Loyalität gegenüber der Staatsführung, die allerdings keineswegs aktiv unterstützt, sondern nur mit groBen Vorbehalten erduldet wurde. Eine größere Öffnung gegenüber dem Nationalsozialismus, die über die nach christlicher Tradition gebotene Gehorsamspflicht der weltlichen Obrigkeit gegenüber hinausgehen sollte, befürworteten in der Pfalz nur sehr wenige ${ }^{75}$ katholische Pfarrer. Die von den Zeitgenossen als „braune“ Pfarrer bezeichneten Geistlichen wurden nicht nur vom nationalsozialistischen Überwachungsapparat als Ausnahme von der Regel wahrgenommen ${ }^{76}$. $\mathrm{Daß}$ sie in der Tat Außenseiter innerhalb des Klerus waren, belegt eine Analyse ihrer Persönlichkeiten. Vor allem der Zölibat erwies sich für einige dieser Pfarrer als kritischer Punkt ihres Selbstverständnisses als katholische Priester ${ }^{77}$. Die daraus

${ }^{73} \mathrm{Vgl}$. Franz Sonnenberger, Der neue „Kulturkampf“. Die Gemeinschaftsschule und ihre historischen Voraussetzungen, in: Bayern in der NS-Zeit, Bd. 3, hrsg. von Martin Broszat u.a. (München, Wien 1981) 235-327.

${ }^{74}$ Dazu vor allem Thomas Breuer, Verordneter Wandel? Der Widerstreit zwischen nationalsozialistischem Herrschaftsanspruch und traditionaler Lebenswelt im Erzbistum Bamberg (VKZG, Reihe B, 60, Mainz 1992).

75 Bei rund 520 Geistlichen, die zwischen 1933 und 1939 in der Diözese Speyer wirkten, konnte der Verfasser 38 Geistliche ermitteln, bei denen sich irgendwelche Hinweise in dieser Richtung ergaben, wobei nach Aktenlage aber nur etwa die Hälfte der Fälle in dem genannten Zusammenhang wirklich interessant sind. Bei 346 ausgewerteten Personalakten aus dem Archiv des Bistums Speyer ergaben sich 26 Fälle, von denen 16 als substantiell zu betrachten sind. - Vgl. auch Priester unter Hitlers Terror, Bd. 1, $101 \mathrm{f}$.

76 Beurteilungen katholischer Priester finden sich u.a. im Landesarchiv Speyer H33/998/ 1000 (Frankenthal), H34/1630 (Germersheim), H37/1873 (Kirchheimbolanden), H38/1349 (Kusel), H38/1398 (Homburg/St. Ingbert), H42/755 (Pirmasens) sowie H91 (Personalakten der Gestapo).

77 In den 346 herangezogenen Personalakten aus dem Archiv des Bistums Speyer fand sich nur bei 22 Geistlichen Material, in dem es in den Jahren zwischen 1933 und 1945 um einen für katholische Priester als ungebührlich empfundenen Umgang mit Frauen ging. Dagegen belegen dies die Akten in 7 der 16 „NS“-Fälle. 
resultierenden Konflikte mit Kollegen und der Bistumsleitung konnten - das gleiche galt für Auseinandersetzungen bei durch Alkoholmißbrauch bedingten Auffälligkeiten - zu einer Annäherung an die NSDAP führen, die weniger weltanschaulich bedingt war, sondern eher darauf abzielte, Druck gegen die Kirchenbehörde auszuüben oder beim Verlust des Pfarramtes eine Beschäftigungsmöglichkeit in einer staatlichen Behörde zu erhalten ${ }^{78}$. Lediglich zwei Geistliche der Diözese Speyer wurden Mitglied der NSDAP; beide schieden aus dem kirchlichen Dienst aus ${ }^{79}$.

War eine öffentliche Unterstützung des Nationalsozialismus durch katholische Kleriker die Ausnahme, so waren die Signale, die von der Spitze der Pfälzischen Landeskirche und von vielen ihrer Pfarrer kamen, andere. Hier gab es eine aktive, zeitweise sogar vorbehaltlose Unterstützung der nationalsozialistischen Regierung. Die in die NSDAP gesetzten Hoffnungen drängten lange Zeit die weitanschaulichen Bedenken in den Hintergrund, die manche Vertreter der NSDAP hervorgerufen hatten, die aber nicht als repräsentativ angesehen worden waren. $\mathrm{Zu}$ einem partiellen Meinungsumschwung kam es erst durch die in der Pfalz mit einiger Verzögerung einsetzenden Auswirkungen der auf Reichsebene heftig geführten kirchenpolitischen Auseinandersetzungen, die nicht zuletzt durch die Einflußnahme der NSDAP auf die Kirche entstanden waren, sowie durch zunehmende neuheidnische Tendenzen in Teilen der Partei bzw. deren Gliederungen. Die Gefährdung der christlichen Glaubenssubstanz durch die weltanschauliche Entwicklung rückte in den Vordergrund, wodurch auch die politische Unterstützung der NSDAP durch viele protestantische Pfarrer in der Pfalz zumindest fragwürdig wurde. Die Gestapo-Akten im Landesarchiv Speyer lassen erkennen, daß der nationalsozialistische Überwachungsapparat zunehmend weniger bereit war, frühere Verdienste um die NSDAP, die einzelnen Pfarrern oder der Landeskirche insgesamt zugeschrieben wurden, zu berücksichtigen ${ }^{80}$. Allerdings wurde diese Entwicklung hin zu einer deutlich schärferen Gangart gegenüber der protestantischen Pfarrerschaft durch den Kriegsbeginn und die Kriegsteilnahme gerade vieler junger Theologen unterbrochen, während sich dies für den katholischen Klerus, der in der Pfalz 1940 erstmals von einer KZ-Einweisung betroffen wurde ${ }^{81}$, nicht generalisierend sagen läßt.

Zusammenfassend kann man feststellen, daß sich in der Zeit zwischen der Saarabstimmung und dem Kriegsbeginn - trotz aller weiterhin feststellbarer konfessioneller Unterschiede - zumindest tendenziell eine Annäherung des Verhältnisses der Pfarrer der beiden großen Kirchen in der Pfalz zum Nationalsozialismus vollzog. Dies gilt sowohl für die Behandlung der Pfarrer durch Staat und Partei als auch für die Wahrnehmung des Nationalsozialismus durch die Pfarrer. Auch evangelische Pfarrer nahmen zunehmend nicht mehr die Übereinstimmung mit

78 Vgl. z. B. Akten im Landesarchiv Speyer H91/1270.

79 Prantl, Geschichte 108 A. 201.

$80 \mathrm{Vgl}$. z. B. Akten im Landesarchiv Speyer H91/4352.

$81 \mathrm{Zu}$ Pfarrer Friedrich Seitz vgl. die Akten im Landesarchiv Speyer H91/6103. 
der Politik der NSDAP in vielen Bereichen als das entscheidende Moment wahr. Vielmehr trat nun die weltanschauliche Auseinandersetzung mit dem Nationalsozialismus in den Vordergrund, dessen Bestrebungen gegen den Einfluß des Christentums in der Gesellschaft nur noch schwerlich als Ausfluß der antikirchlichen Haltung einzelner Parteifunktionäre interpretiert werden konnten.

$\mathrm{Zu}$ einer Zusammenarbeit von Pfarrern beider Konfessionen führte diese Erkenntnis jedoch nicht, auch wenn es vereinzelt zu Absprachen der beiden Kirchenleitungen in Speyer kam. Die Gräben zwischen den Konfessionen waren zu tief, die Konzentration auf die jeweiligen Eigeninteressen zu stark. Ein besseres Verhältnis der Konfessionen entwickelte sich erst nach 1945 - nicht zuletzt aufgrund der Erfahrungen der NS-Zeit ${ }^{82}$.

82 Allerdings müßte noch näher untersucht werden, inwieweit ein besseres Verhältnis der Konfessionen, wie es etwa durch den Verzicht auf eine Wiederbelebung des Zentrums und die Gründung der CDU zum Ausdruck kam, tatsächlich als charakteristisch für die unmittelbare Nachkriegszeit anzusehen ist. 